\title{
High Resolution Computed Tomography Features of Third Wave of COVID-19 Pneumonia in Confirmed Cases
}

\author{
Abdul Malik Hayat ${ }^{1}$, Shahzad Karim Bhatti ${ }^{2}$ \\ ${ }^{1}$ Department of Radiology, Sir Ganga Ram Hospital, Lahore, Pakistan \\ ${ }^{2}$ Department of Radiology, King Edward Medical University, Lahore, Pakistan \\ Email: malikhayat@yahoo.com, Shahzadkbhatti@gmail.com
}

How to cite this paper: Hayat, A.M. and Bhatti, S.K. (2021) High Resolution Computed Tomography Features of Third Wave of COVID-19 Pneumonia in Confirmed Cases. Advances in Computed Tomography, 10, 11-17.

https://doi.org/10.4236/act.2021.102002

Received: May 9, 2021

Accepted: June 27, 2021

Published: June 30, 2021

Copyright ( 2021 by author(s) and Scientific Research Publishing Inc. This work is licensed under the Creative Commons Attribution International License (CC BY 4.0).

http://creativecommons.org/licenses/by/4.0/

\section{(c) (i) Open Access}

\begin{abstract}
COVID-19 which is caused by its new type called SARS-CoV-2 is a viral disease predominantly involving the lungs. Objective: To investigate HRCT features of pulmonary disease in COVID-19 in Lahore, Pakistan. Methods: This is a prospective study that involved 127 COVID-19 positive patients (age 18 80 years, both genders) through non-probability sampling was conducted at the Radiology Department, Sir Ganga Ram Hospital, Lahore, in 2021. All patients with RT-PCR positive underwent HRCT chest. All findings in HRCT chest were assessed. Confirmed patients had positive HRCT. Excluded situations are low quality of images irrespective of its reason, HRCT indications other than COVID-19 pneumonia, and patients who do not want to participate in the study Results: Considering the exclusion and inclusion criteria, totally 127 COVID-19 confirmed patients ranging age from 18 to 80 years with a mean age of $52 \pm 18$ years, took part in this study. The most important and common HRCT finding was the multilobar ground-glass pattern which was present in $95 \%$ of patients. Other findings including, crazy paving pattern, consolidation, air bronchogram, and bronchiectasis were present in $8.7 \%, 82 \%$, $63 \%$, and $37 \%$ of patients respectively. Pleural effusion seen in $21 \%$ patients. $16 \%$ of patients had mediastinal lymphadenopathy. Conclusion: In our study, the ground-glass pattern was found to be the most common and important HRCT finding in patients confirmed with COVID-19 pneumonia. This important HRCT pattern is mostly found to be in posterobasal and peripheral subpleural locations. Other than ground-glass pattern, bronchiectasis, and consolidation having the air bronchogram were also reported commonly.
\end{abstract}

\section{Keywords}

High Resolution Computed Tomography (HRCT), COVID-19, Ground-Glass Pattern, Third Wave 


\section{Introduction}

Covid-19 which is caused by SARS-CoV-2 erupted for the first time in Wuhan city of China in Dec, 2019 [1]. This virus mainly affects the lungs, resulting in mild to severe pneumonia which took the lives of millions of people around the world. Coronavirus has different variants including the newly identified UK variant (B.1.1.7) in December 2020 [2]. Since then other new variants have also appeared in other locations around the world. In Pakistan it was first isolated in Feb 2021, which has caused severe pneumonia in comparison with the previous strain.

However the Real-time polymerase chain reaction (RT-PCR) is considered the gold standard for the diagnosis of COVID-19, there are some limitations to this lab test as well, which include, probability of false negativity [3] [4], it is timeconsuming and not readily available, as well as low sensitivity of about $60 \%$ $71 \%$ [5]. HRCT (high-resolution CT) has a crucial role in the diagnosis of COVID-19 pneumonia with a sensitivity of up to $98 \%$ [6], particularly in cases with negative PCR or asymptomatic patients. HRCT is also useful in the assessment of the degree of damage [7] and monitoring the progression of the disease process.

Despite a wide variety of HRCT findings of COVID-19 pneumonia including crazy paving pattern, reversible halo sign, reticular pattern, airway changes [8] [9] [10], ground-glass opacity (GGO) with posterior and peripheral distribution [11] was found to be the hallmark. This is also confirmed by the recent study performed by Omar et al. [5].

After a review of the role of imaging in COVID-19 in recent studies, our objective is to study the HRCT features of COVID-19 pneumonia.

\section{Methods}

This prospective study which includes 127 COVID-19 positive patients (age 18 80 years, both genders) was conducted at Radiology Department of Sir Ganga Ram Hospital, Lahore in 2021 after clearance of local ethical review board.

Confirmed patients with brief clinical history were referred to radiology department during a time period of March 15 to April 15, 2021 for taking HRCT. After taking informed consent from the patients or their attendants and excluding situations like the low quality of images irrespective of its reason, HRCT indications other than COVID-19 pneumonia and patients who do not want to participate in the study, HRCT chest images without IV contrast were obtained in supine position in end-inspiratory phase. The 4-slice TOSHIBA CT scan machine was used to take all images with $2 \mathrm{~mm}$ slice thickness using $120 \mathrm{KV}$ and $300 \mathrm{mAs}$. Bone algorithm was used for reconstruction process. As CT machine was being used for COVID-19 patients. After each scan CT machine was disinfected with ethanol. Data is then transferred to the workstation for interpretation. All variables including continuous and discrete were described as medians/ranges, and counts/percentages respectively. 


\section{Results}

Considering the exclusion and inclusion criteria which were already mentioned in method section, totally 127 COVID-19 confirmed patients ranging age from 18 to 80 years with mean age of $52 \pm 18$ years, took part in this study. There were 74 male and 53 female patients with a male to female ratio of 1.2:1. Demographic and major clinical characteristic are stated in Table 1. All HRCTs were interpreted and various CT features in COVID-19 infection were assessed. The pattern of detected HRCT findings is described in Table 2 and shown in Figures 1-5. Ground-glass haze was seen in all patients (100\%) as shown in Figures 1-5. 119 patients $(93.7 \%)$ revealed bilateral lung involvement as shown in Figures 1-3 and Figure 5. Only one patient had unilateral involvement (0.78\%) as shown in Figure 4. Postero-basal and peripheral subpleural distribution of ground-glass haze were observed in 121 (95.2\%) and 115 (90.5\%) patients respectively with subpleural predominance shown in Figure 1 and Figure 5. Multi-lobe involvement was present in 113 (88.9\%) patients with basal predominance shown in Figure 2, Figure 3 and Figure 5. Upper lobe distribution, consolidation, air bronchogram, bronchiectasis, crazy paving pattern, pleural effusion and mediastinal lymphadenopathy were identified in 78 (61.4\%), 104 (81.8\%), 80 (63\%), 46 (36.2\%), 11 (8.6\%), $127(3.1 \%)$ and $19(14.9 \% \%)$ patients respectively. whereas septal thickening was

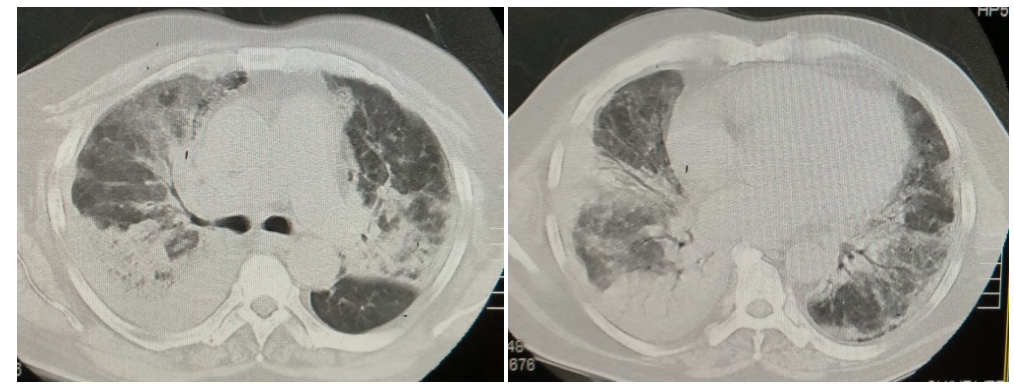

Figure 1. A 44-year-old male who presented with cough and shortness of breath, HRCT illustrates bilateral ground-glass opacities and lower lobe consolidation with air bronchogram along with right sided mild effusion.

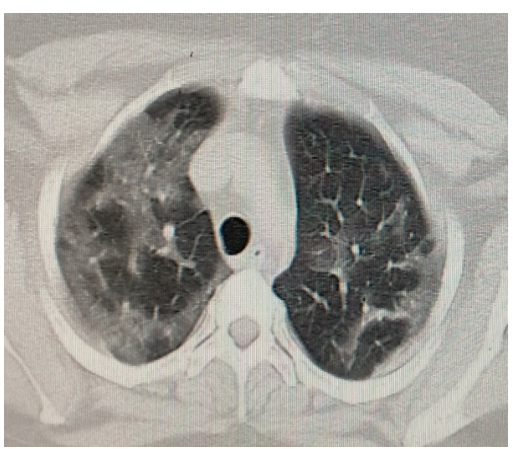

(a)

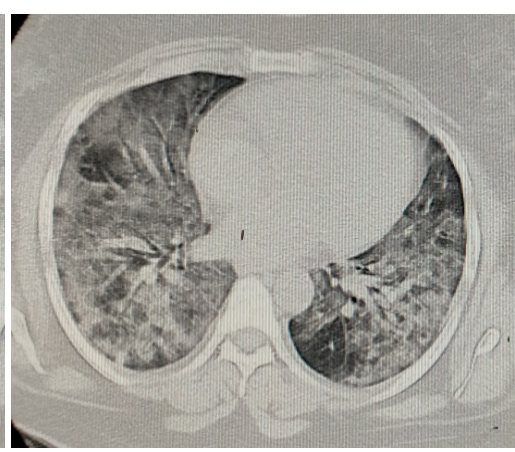

(b)

Figure 2. A 56-year-old female presented with fever, cough and dyspnea. HRCT demonstrates (a) bilateral predominantly peripherally based ground-glass opacities, and (b) Multilobar, diffuse, bilateral, confluent ground-glass opacities with crazy paving pattern and consolidation. 


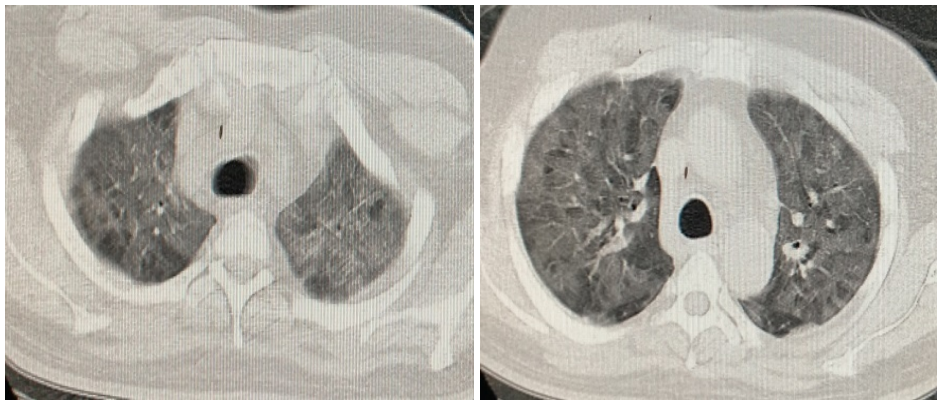

Figure 3. A 48 years old male who presented with severe dyspnea, HRCT illustrates multiple ground-glass haze bilaterally with upper lobe predominance.

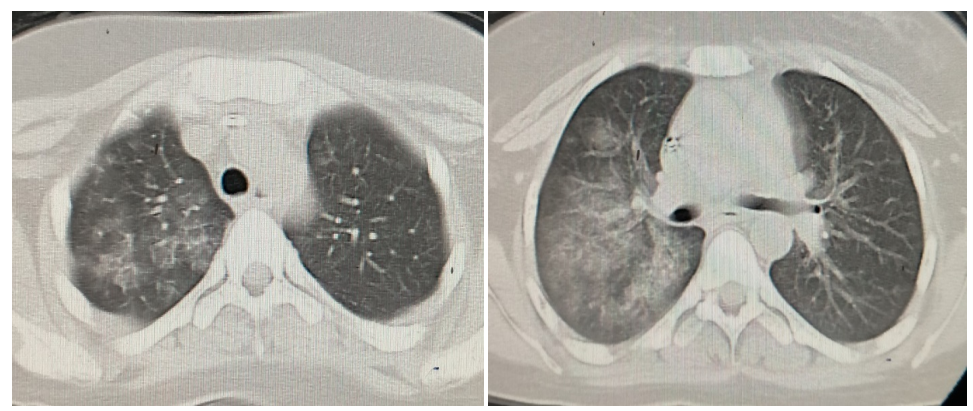

Figure 4. Female, HRCT illustrates right lung multiple ground-glass and consolidating opacities with right upper lobe predominance.

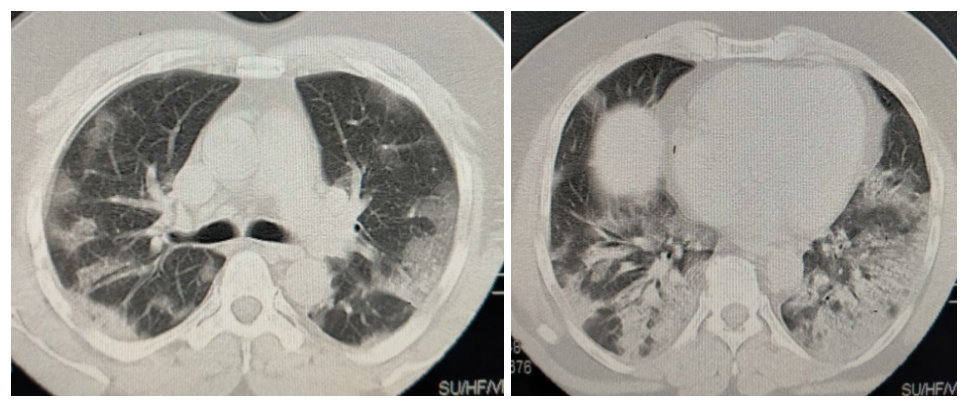

Figure 5. HRCT of 52-year-old male illustrates multiple, peripheral groundglass and consolidating opacities predominantly posterior in distribution with multi-lobar involvement.

Table 1. Demographic and major clinical characteristics.

\begin{tabular}{|c|c|c|}
\hline Characteristic & Group & Number of patients (\%) \\
\hline Gender & $\begin{array}{l}\text { - Male } \\
\text { - female }\end{array}$ & $\begin{array}{l}\text { - } 74(58.26 \%) \\
\text { - } 53(42.51 \%)\end{array}$ \\
\hline Age & $\begin{array}{l}\text { - } 18 \text { - } 29 \\
\text { - } 30-39 \\
\text { - } 40-49 \\
\text { - } 50-59 \\
\text { - }>60\end{array}$ & $\begin{array}{ll}\text { - } & 0(0 \%) \\
\text { - } & 2(1.57 \%) \\
\text { - } & 41(32.28 \%) \\
\text { - } & 56(44.09 \%) \\
\text { - } & 28(22.04 \%)\end{array}$ \\
\hline Major Clinical data & $\begin{array}{l}\text { - Fever } \\
\text { - Dry cough } \\
\text { - Tiredness } \\
\text { - SOB }\end{array}$ & $\begin{array}{l}\text { - } 127(100 \%) \\
\text { - } 114(89.76 \%) \\
\text { - } 127(100 \%) \\
\text { - } 103(81.10 \%)\end{array}$ \\
\hline
\end{tabular}


Table 2. HRCT features in confirmed patients of COVID-19 pneumonia.

\begin{tabular}{cc}
\hline CT features & Number of cases (\%) \\
\hline Ground-glass opacity & $127(100 \%)$ \\
Bilateral involvement & $119(93.7 \%)$ \\
Unilateral involvement & $1(0.78 \%)$ \\
Peripheral distribution & $121(95.2 \%)$ \\
Posterior distribution & $115(90.5 \%)$ \\
Multilobar and basal involvement & $113(88.9 \%)$ \\
Upper lobe involvement & $78(61.4 \%)$ \\
Consolidation & $104(81.8 \%)$ \\
Air bronchogram & $80(63 \%)$ \\
Bronchiectasis & $46(36.2 \%)$ \\
Crazy paving pattern & $11(8.6 \%$ \\
Pleural effusion & $4(3.1 \%)$ \\
Mediastinal lymphadenopathy & $19(14.9 \%)$ \\
Pulmonary nodule & $2(1.5 \%)$ \\
\hline
\end{tabular}

appreciated in less than $20 \%$ of patients. Only two patients had pulmonary nodules. Consolidation with air bronchogram is shown in Figure 1, however crazy paving is shown in Figure 2.

Commonly observed HRCT findings are shown image vise above.

\section{Discussion}

However, lots of studies conducted to evaluate findings of COVID-19 pneumonia in HRCT chest so far, particularly in the last year. We wanted to perform a study as well, in order to evaluate findings of disease during the third wave of COVID-19 in our population in 2021. Among 127 confirmed cases, the most common feature in all patients was found to be ground-glass opacification. Most of the hazes were observed bilaterally with typical posterior and peripheral distribution. Other typical manifestations were included consolidation having air bronchogram, bronchiectasis and crazy paving. Less common features were pleural effusion and septal thickening. Pulmonary nodules also presented as atypical features.

In comparison with our study, research conducted by Zhu et al. reported that ground-glass opacification was appreciated in only $47 \%$ of patients [12] [13]. As well, we described that consolidation presented in $81.8 \%$ of patients, however Salehi et al. [14] who conducted research on 919 patients reported that only $31 \%$ of patients had consolidation and prevalence of Peripheral and bilateral involvement of lungs and ground-glass opacification were reported as of (95.2\% vs $76 \%)$ and $(100 \%$ vs $88 \%)$ respectively. Study done by Omer et al. [6] which is performed on 30 patients, showing that crazy paving as compared to our study 
was found in $13.3 \%$ of cases. Mediastinal lymphadenopathy was not a common finding in our study.

A study by Bai et al. reported that $59 \%$ confirmed patients had increased calibre of pulmonary vessels at subsegmental level [15] [16]. This unusual finding was also reported by Albarello et al. in only 2 patients [17]. However, we did not report any subsegmental pulmonary vessel dilatation in any of our patients. Although the inflammatory changes have been shown to be the cause of pulmonary vessel dilatation [18], variable studies attribute hyperemia caused by COVID19 as compared to other viral pulmonary diseases [19] [20] [21].

Despite constant referral of COVID-19 confirmed patients, there were few limitations too, including small sample size, follow up and almost lack of pediatric cases as well as no histopathologic correlation.

\section{Conclusion}

HRCT chest plays an important role in the diagnosis of COVID-19 pneumonia initially and can help in the proper assessment of the severity of the infectious process. In our study, the ground-glass haze was found to be the most common findings in all patients. Most of the hazes were observed bilaterally with the typical posterior and peripheral subpleural distribution involving multiple lobes, predominantly basal. Consolidation associated with air bronchogram and bronchiectasis was also found commonly.

\section{Conflicts of Interest}

The authors declare no conflicts of interest regarding the publication of this paper.

\section{References}

[1] Zhu, N., Zhang, D., Wang, W., et al. (2020) A Novel Coronavirus from Patients with Pneumonia in China, 2019. The New England Journal of Medicine, 382, 727-733. https://doi.org/10.1056/NEJMoa2001017

[2] Wise, J. (2020) Covid-19: New Coronavirus Variant is Identified in UK. BMJ, 371, Article No. m4857. https://doi.org/10.1136/bmj.m4857

[3] Xie, X., Zhong, Z., Zhao, W., Zheng, C., Wang, F. and Liu, J. (2020) Chest CT for Typical 2019 (Covid-19) Pneumonia: Relationship to Negative RT-PCR Testing. Radiology, 296, E41-E45. https://doi.org/10.1148/radiol.2020200343

[4] Huang, P., Liu, T., Huang, L., et al. (2020) Use of Chest CT in Combination with Negative RT-PCR Assay for the 2019 Novel Coronavirus but High Clinical Suspicion. Radiology, 295, 22-23. https://doi.org/10.1148/radiol.2020200330

[5] Omar, et al. (2020) High-Resolution CT Features of COVID-19 Pneumonia in Confirmed Cases. Egyptian Journal of Radiology and Nuclear Medicine, 51, Article No. 121. https://doi.org/10.1186/s43055-020-00236-9

[6] Fang, Y., Zhang, H., Xie, J., et al. (2020) Sensitivity of Chest CT for COVID-19: Comparison to RT-PCR. Radiology, 296, E115-E117. https://doi.org/10.1148/radiol.2020200432

[7] National Health Commission of the People's Republic of China (2020) The Diag- 
nostic and Treatment Protocol of COVID-19. http://www.gov.cn/zhengce/zhengceku/2020-02/19/content_5480948.htm

[8] Fang, Y., Zhang, H., Xu, Y., Xie, J., Pang, P. and Ji, W. (2020) CT Manifestations of Two Cases of 2019 Novel Coronavirus (2019-nCoV) Pneumonia. Radiology, 295, 208-209. https://doi.org/10.1148/radiol.2020200280

[9] Qian, L., Yu, J. and Shi, H. (2020) Severe Acute Respiratory Disease in a Huanan Seafood Market Worker: Images of an Early Casualty. Radiology. Cardiothoracic Imaging, 14, e200033. https://doi.org/10.1148/ryct.2020200033

[10] Bernheim, A., Mei, X., Huang, M., et al. (2020) Chest CT Findings in Coronavirus Disease-19 (COVID-19): Relationship to Duration of Infection. Radiology, 295, 685-691. https://doi.org/10.1148/radiol.2020200463

[11] Wang, D., Hu, B., Hu, C., et al. (2020) Clinical Characteristics of 138 Hospitalized Patients with 2019 Novel Coronavirus-Infected Pneumonia in Wuhan, China. The Journal of the American Medical Association, 323, 1061-1069. https://doi.org/10.1001/jama.2020.1585

[12] Zhu, W., Xie, K., Lu, H., Xu, L., Fang, S. and Zhou, S. (2020) Initial Clinical Features of Suspected Coronavirus Disease 2019 in Two Emergency Departments outside of Hubei, China. Journal of Medical Virology, 92, 1525-1532. https://doi.org/10.1002/jmv.25763

[13] Albarello, F., Pianura, E., Di Stefano, F., et al. (2020) 2019-Novel Coronavirus Severe Adult Respiratory Distress Syndrome in Two Cases in Italy: An Uncommon Radiological Presentation. International Journal of Infectious Diseases, 93, 192-197. https://doi.org/10.1016/j.ijid.2020.02.043

[14] Salehi, S., Abedi, A., Balakrishnan, S. and Gholamrezanezhad, A. (2020) Coronavirus Disease 2019 (COVID-19): A Systematic Review of Imaging Findings in $919 \mathrm{~Pa}$ tients. American Journal of Roentgenology, 215, 87-93. https://doi.org/10.2214/AJR.20.23034

[15] Kay, F. and Abbara, S. (2020) The Many Faces of COVID-19: Spectrum of Imaging Manifestations. Radiology: Cardiothoracic Imaging, 2, e200037. https://doi.org/10.1148/ryct.2020200037

[16] Chung, M., Bernheim, A., Mei, X., et al. (2020) CT Imaging Features of 2019 Novel Coronavirus (2019-nCoV). Radiology, 295, 202-207. https://doi.org/10.1148/radiol.2020200230

[17] Bai, H.X., Hsieh, B., Xiong, Z., et al. (2020) Performance of Radiologists in Differentiating COVID-19 from Viral Pneumonia on Chest CT. Radiology, 296, E46-E54. https://doi.org/10.1148/radiol.2020200823

[18] Ye, Z., Zhang, Y., Wang, Y., et al. (2020) Chest CT Manifestations of New Coronavirus Disease 2019 (COVID-19): A Pictorial Review. European Radiology, 30, 43814389. https://doi.org/10.1007/s00330-020-06801-0

[19] Li, W., Moore, M.J., Vasilieva, N., et al. (2003) Angiotensinconverting Enzyme 2 Is a Functional Receptor for the SARS Coronavirus. Nature, 426, 450-454. https://doi.org/10.1038/nature02145

[20] Nicolaou, S., Al-Nakshabandi, N.A. and Muller, N.L. (2003) SARS: Imaging of Severe Acute Respiratory Syndrome. American Journal of Roentgenology, 180, 1247-1249. https://doi.org/10.2214/ajr.180.5.1801247

[21] Ooi, G.C., Khong, P.L., Muller, N.L., et al. (2004) Severe Acute Respiratory Syndrome: Temporal Lung Changes at Thinsection CT in 30 Patients. Radiology, 230, 836-844. https://doi.org/10.1148/radiol.2303030853 